\title{
Origen de epónimos comúnmente empleados en la Unidad de Cuidados Intensivos
}

\section{Origin of commonly used eponyms in the Intensive Care Unit daily job}

\author{
Alejandro Donoso F. ${ }^{a}$, Daniela Arriagada S. ${ }^{\text {a }}$, Megan Neumann B. ${ }^{\text {b }}$ \\ aMédico. Unidad de Paciente Crítico Pediátrico. Hospital Clínico Metropolitano La Florida. Santiago, Chile

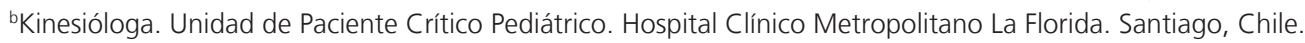 \\ Alumna de Medicina Universidad Andrés Bello
}

Recibido el 9 de enero de 2018, aceptado el 29 de enero de 2018

\section{Resumen}

Los epónimos reflejan la historia de la medicina, las enfermedades y los médicos en su época. Su uso es controversial, presentando partidarios y detractores. No obstante, el empleo de epónimos persiste en el lenguaje médico contemporáneo en las Unidades de Cuidados Intensivos e incluyen a algunos de frecuente uso como: Foley, Seldinger, Down, Macintosh, Magill, Ringer, Yankauer, Doppler y French. El objetivo de la presente revisión es fomentar el conocimiento biográfico y la época histórica en la cual tomó lugar su quehacer médico o laboral, para así profundizar aspectos de la historia de la medicina.

\footnotetext{
Abstract

Eponyms reflect the history of medicine, diseases, and doctors in their time. Their use is controversial, presenting supporters and detractors. However, the use of eponyms persist in the modern medical language in the Intensive Care Units and includes some frequently used ones such as Foley, Seldinger, Down, Macintosh, Magill, Ringer, Yankauer, Doppler, and French. The objective of this review is to promote biographical knowledge and the historical period in which its medical use took place, in order to deepen aspects of medicine history.
}

Palabras clave:

Cuidados Intensivos;

biografía;

epónimos;

etimología;

historia de la medicina
Keywords:

Intensive Care;

biography;

eponyms;

etymology;

medicine history 


\section{Introducción}

El uso de epónimos es frecuente en el lenguaje médico, siendo parte de nuestra tradición y cultura histórica ${ }^{1}$. Muchos de ellos han perdurado hasta la actualidad $^{2} \mathrm{y}$, aunque su empleo presenta detractores ${ }^{3}$, su utilización sigue estando arraigada en la práctica clínica cotidiana de las Unidades de Cuidados Intensivos (UCI $)^{4}$ (figura 1). Sin embargo, por quienes los emplean, existe escaso conocimiento sobre las personas detrás del epónimo.

La mayoría de los epónimos se originaron a finales del siglo XIX e inicios del siglo XX ${ }^{5}$ reflejando el dominio de la cultura científica y lenguaje en aquel entonces $y$ han dado nombre a quienes identificaron, describieron o publicaron por primera vez enfermedades, estructuras anatómicas, fenómenos fisiológicos o físicos, síndromes, signos de enfermedades, medicamentos, técnicas, dispositivos, procedimientos, etc. ${ }^{6}$. En la actualidad, no es habitual que surjan epónimos dado que el desarrollo de la medicina es el resultado del trabajo en equipo multidisciplinario.

A continuación, se revisa en forma breve la biografía de algunas personas que dieron origen a epónimos de uso clásico en UCI (figura 2), con el objetivo de fomentar el conocimiento biográfico y la época histórica en la cual tomó lugar su quehacer médico o laboral, para así profundizar aspectos de la historia de la medicina. No se realizó búsqueda sistemática y se excluyeron nombres de enfermedades y signos clínicos.

\section{Foley, Frederic Eugene Basil (1891-1966)}

Nació en St. Cloud, Minnesota, Estados Unidos en el año 1891. Durante su juventud estudió retórica en la Universidad de Yale y se desempeñó como profesor de inglés en una escuela de preparatoria. Sin embargo, su interés de larga data por la ciencia lo llevó a estudiar en la Escuela de Medicina de la Universidad Johns Hopkins, graduándose en el año 1918. Durante el primer año de postgrado, se dedicó a la anatomía patológica y luego recibió entrenamiento quirúrgico bajo la supervisión de William Halsted y Harvey Cushing, reconociendo a este último como su mentor, lo que motivó decididamente a Foley a ser neurocirujano. Lamentablemente, no contó con el apoyo de su mentor, debiendo considerar otras especialidades quirúrgicas, regresando así a Minnesota donde daría inicio a su labor como urólogo en el Hospital St. Paul-Ramsey ${ }^{7}$. Aunque no tuvo un entrenamiento formal, recibió su certificación de urólogo en el año 1937. Cabe destacar que ocho años antes ya había diseñado una sonda con balón destinado a la hemostasia de cirugía prostática ${ }^{8}$. Posteriormente, aprovechó el avance en la tecnología del látex enfocándose en el desarrollo de una sonda destinada al drenaje continuo de la orina de los pacientes, para lo cual se basó en el balón previamente diseñado con fin hemostático para la fijación de dicha sonda. Así, ideó la sonda vesical de doble lumen (como unidad única), presentándola en 1935 ante la Sociedad Americana de Urología y publicando su nueva sonda

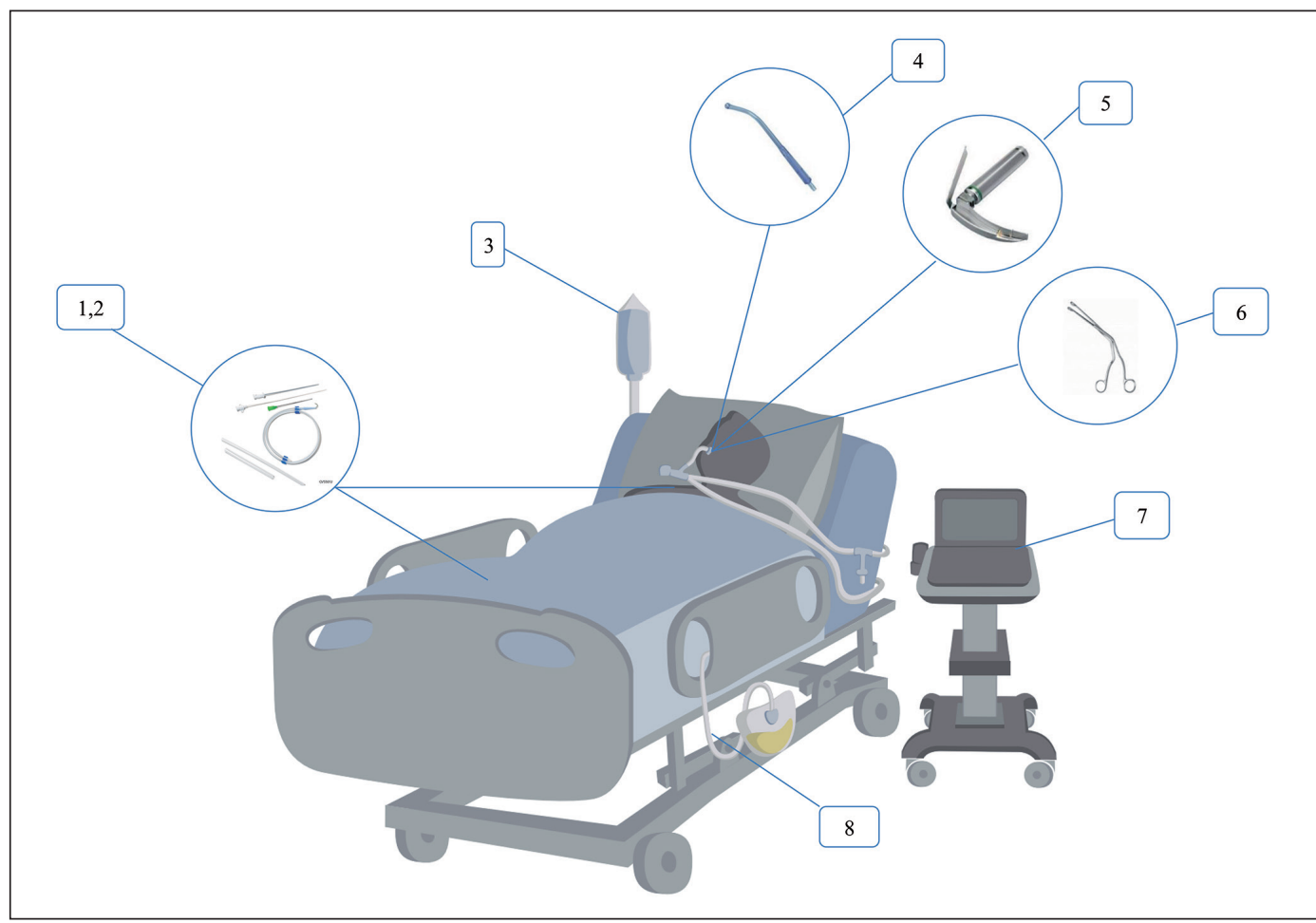

Figura 1. Ejemplos de diversos epónimos utilizados en la atención de un paciente críticamente enfermo. 1) Técnica/Catéter Seldinger; 2) Unidad de medida French; 3) Solución Ringer; 4) Yankauer; 5) Hoja Macintosh; 6) Pinza Magill; 7) Ecografía Doppler; 8) Sonda Foley. 

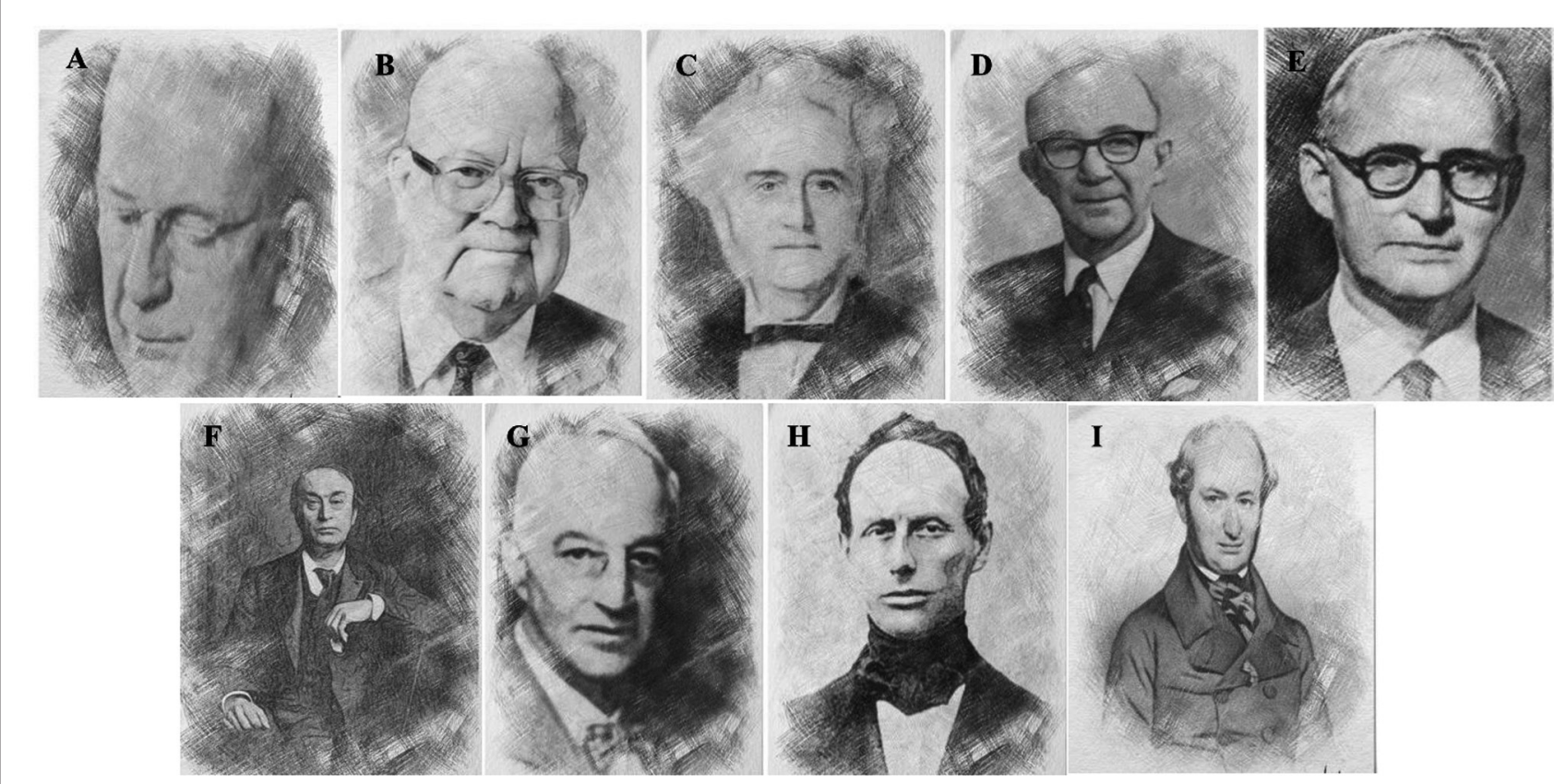

Figura 2. Célebres personas que dieron origen a epónimos de uso frecuente en medicina intensiva infantil. A) Frederic Foley (1891-1966). Sonda urinaria. B) Sven-Ivar Seldinger (1921-1998). Técnica de cateterización. C) John Down (1828-1896). Descripción fenotípica de los pacientes con síndrome de Down. D) Robert Reynolds Macintosh (1897-1989). Hoja curva de laringoscopio. E) Ivan Magill (1888-1986). Pinza utilizada para intubación. F) Sidney Ringer (1835-1910). Solución intravenosa. G) Sidney Yankauer (1872-1932). Cánula rígida para aspiración. H) Christian Doppler (1803-1853). Fenómeno físico. I) Joseph-Frédéric-Benoît Charrière (1803-1876). Escala francesa de calibre.

en el Journal of Urology en $1937^{9}$. No obstante, la patente comercial inicialmente no quedó a nombre del Dr. Foley por disputas con la casa Davol Rubber Company las cuales se originaron debido a que el Dr. Foley no pudo demostrar fehacientemente que fue él su inventor original. El motivo por el cual la sonda siguió llamándose Foley, a pesar de haber perdido la batalla legal por la patente, se debe a que la C.R. Bard Company de New Jersey ya había empezado a comercializar los catéteres antes de la primera patente, en 1935, con el nombre de catéter de Foley.

El Dr. Foley asimismo desarrolló técnicas quirúrgicas (plastia ureteropélvica) ${ }^{10} \mathrm{e}$ instrumental para el quirófano (resectoscopio) $)^{11}$.

En la actualidad, la sonda Foley originada como solución en la búsqueda de una mejor atención en la práctica clínica, es prácticamente la misma que diseñó Frederic Foley hace casi un siglo.

\section{Seldinger, Sven-Ivar (1921-1998)}

El Dr. Seldinger, proveniente de una familia de mecánicos y relojeros, nació en Mora, Suecia en 1921. Estudió medicina en el Instituto Karolinska de Estocolmo, donde se licenció en 1948.

En 1950 inició la residencia en Radiología y fue allí, siendo aún residente de primer año, donde ideó la téc- nica que llevaría su nombre. Tras intentar introducir, sin éxito, un catéter preformado largo y flexible a través de una punción arterial, se encontró con tres objetos en su mano: una aguja, un alambre y un catéter. Y, según su propio relato, se dio cuenta, en fracción de segundos del orden en que debería utilizarlos: "needle in-wire in-needle off-catheter on wire-catheter in-wire off'. La técnica fue publicada en Acta Radiológica en $1953^{12}$ y utilizada por primera vez en la ubicación de un adenoma paratiroideo ${ }^{13}$.

No obstante, su jefe en el Instituto Karolinska no consideró que esta idea tuviera el interés suficiente como para justificar una tesis doctoral. Así, Seldinger tuvo que empezar un nuevo proyecto, esta vez utilizando su técnica percutánea para cateterizar la vía biliar. Finalmente, se doctoró en 1966 con un trabajo sobre la colangiografía transparietohepática ${ }^{14}$. En 1967, pasó a dirigir el Departamento de Radiología del hospital de su ciudad natal. Allí murió en el año 1998.

Se dice que el Dr. Seldinger atribuyó su técnica de cateterización percutánea a un momento de un "grave ataque de sentido común".

\section{Down, John Langdon (1828-1896)}

El Dr. Down nació en 1828 en la sureña localidad inglesa de Torpoint, un pueblo de clase obrera, dentro 
de una familia de origen irlandés, viviendo entre artesanos. Fue a la escuela hasta los catorce años para luego ayudar en la tienda de su padre. A los 18 años ocurrió un episodio significativo en su vida, el cual marcaría su futuro profesional. Down relató lo siguiente al momento de refugiarse con su famila de una lluvia estival dentro de una cabaña: "I was brought into contact with a feeble minded girl, who waited on our party and for whom the question haunted me - could nothing for her be done? I had then not entered on a medical student's career but ever and anon... the remembrance of that hapless girl".

Su primer paso hacia la carrera de medicina se inició en Londres donde se desempeñó como aprendiz de cirujano, pero necesitando una formación más sólida se inscribió en curso de ciencias básicas en la Real Sociedad Farmacéutica donde destacó académicamente. Finalmente, ingresó a estudiar medicina a los 25 años en el London Hospital, donde fue un estudiante brillante. Al poco tiempo de graduado fue nombrado superintendente médico en el Real Asilo de Earlswood, donde a partir de su conocimiento de la antropología física de Johan Frederich Blumenbach, se empeñó en asignar a todos los residentes del asilo, mediante fotografías que él mismo tomaba, a algún grupo racial, basándose en el diámetro del cráneo y características faciales específicas; pero, su clasificación étnica no fue ampliamente aceptada y el mismo la abandonó al debido tiempo ${ }^{15}$. Su descripción del grupo Mongoliano, llevó al específico reconocimiento de estos residentes como una categoría distinta, publicando su observación en $1866^{16}$ en la revista London Hospital Reports, en donde relata: "El pelo no es negro, como en el Mongol verdadero, sino de un color pardusco, lacio y escaso. La cara es plana y amplia. Las mejillas son rotundas y se extienden a los lados. Los ojos se colocan oblicuamente, y su canto interno es más distante de lo normal. La grieta palpebral es muy estrecha. La frente se arruga transversalmente con la ayuda constante de los elevadores del párpado. Los labios son grandes y gruesos, con grietas transversales. La lengua es larga y áspera. La nariz es pequeña. La piel tiene un leve tinte amarillento sucio, y es deficiente en elasticidad, dando el aspecto de ser demasiado grande para el cuerpo".

Casi un siglo después un grupo de connotados genetistas escribieron una carta a The Lancet donde proponen abandonar este término ${ }^{17}$. Lo cual fue corroborado en 1965 por la Organización Mundial de la Salud.

El Dr. Down era de ideas liberales. Defendió que las mujeres podían acceder a todas las profesiones y debían tener derecho a voto. Se opuso fuertemente a la esclavitud. Igualmente, estimuló una educación especial y el dar oportunidades a todos los niños discapacitados.
En 1890 contrajo una grave influenza, tras la cual dejó de ejercer como médico. Murió en el año 1896.

\section{Macintosh, sir Robert Reynolds (1897-1989)}

Nació en Timaru, Nueva Zelanda. Siendo bautizado con el nombre maorí de Rewi Rawhiti. A los 18 años se incorporó a la Royal Flying Corps de la British Army sirviendo como piloto en la primera guerra mundial. En 1917 su avión fue derribado en Francia y tomado prisionero, logrando escapar y regresar al Reino Unido, donde inició sus estudios de Medicina. Su interés era ser cirujano, no obstante, empezó a dar anestesia en clínicas dentales y quirúrgicas. Se graduó como médico el año 1924 y prontamente se dio cuenta de la necesidad de anestesistas expertos, comenzando una fructífera y reconocida práctica anestésica en Londres llegando a ser profesor de anestesia en la Universidad de Oxford en $1937^{18}$.

Como muchos inventos en medicina, su diseño del laringoscopio nació de la necesidad. Los médicos británicos en los campos de batalla de la Segunda Guerra Mundial querían una hoja que no tocara la epiglotis, para que pudieran intubar las tráqueas de víctimas semiinconscientes. Así, en el año 1943 diseñó una elegante y sencilla hoja de laringoscopio curva la cual permitía levantar directamente la epiglotis en vez de retraerla ${ }^{19}$, accediendo así en forma segura a la vía aérea. Macintosh basó su diseño en la mordaza de BoyleDavis que él frecuentemente usaba cuando asistía las amigdalectomías.

El aporte de Macintosh no fue solo la forma de la hoja, sino que también la técnica utilizada (ubicar la punta de la hoja en la vallécula epiglótica para permitir la exposición entera de la laringe ${ }^{20}$ lo cual también originaba menor necesidad de sedación e incidencia de laringoespasmo ${ }^{21}$.

A pesar de su experiencia traumática militar, prestó ayuda como médico en la Segunda Guerra Mundial en la Royal Air Force, donde diseñó un nuevo modelo de los chalecos salvavidas de los pilotos ${ }^{18}$.

El Dr. Macintosh siempre predicó sobre una anestesia "segura y simple". En el año 1949 impulsó el desarrollo de un comité para investigar las muertes asociadas a anestesia, idea que en un inicio no fue bien recibida por la comunidad médica.

En la actualidad, el laringoscopio Macintosh es el más popular ${ }^{22}$.

\section{Magill, Ivan Whiteside (1888-1986)}

Nació en 1888 en Irlanda del Norte. Se graduó de médico en la Queen's University en 1913, donde le expidieron un certificado confirmando que durante la 
carrera había recibido formación práctica en uso de anestésicos, habiendo administrado sólo "una anestesia" en el Royal Victory Hospital de Belfast ${ }^{23}$.

Con el inicio de la Gran Guerra, tomó una comisión en el Real Cuerpo Médico del ejército, donde se desempeñó hasta 1919. Ya terminado el conflicto bélico, fue a trabajar con Harold Gillies, un ilustre cirujano maxilofacial del Queen's Hospital, en Kent. Este recinto era especializado en lesiones faciales, en el cual Magill trabajó como anestesista.

En ese entonces, la anestesia consistía en una mezcla de aire y éter que se administraban a través de una especie de cánula faríngea de goma. Este sistema suponía varios problemas: la vía aérea no estaba aislada y, además, no se podría vencer una obstrucción de la misma en el caso de producirse; pero el mayor inconveniente era que el cirujano se exponía a una dosis no despreciable de anestésicos. En 1922, casualmente, mientras Magill estaba intentando anestesiar a un soldado con una grave deformación mandibular se encontró con que el catéter faríngeo no permitía una correcta espiración, por lo que el paciente no podía ser adecuadamente ventilado, ante esto Magill colocó un segundo catéter por vía nasal que llegó directamente hasta la tráquea, mejorando significativamente la ventilación y permitiendo que la espiración del gas anestésico se realizara por el interior del tubo traqueal ${ }^{24}$.

La intubación nasal a ciegas fue desarrollada por primera vez y descrita por Magill, en $1928^{25}$. Esta consistía en la inserción de un tubo biselado de goma en un paciente en "posición de olfateo". Guiado solamente por los sonidos respiratorios, era capaz de intubar a ciegas a través de la nariz y confirmar la correcta posición del tubo.

Paralelamente al desarrollo de las técnicas anestésicas, también diseñó los aparatos e instrumentos necesarios para ello. La primera publicación de Magill, en 1920, fue la descripción de un fórceps para guiar el tubo nasotraqueal en la laringe, el que actualmente es conocido como "pinzas de Magill". Este dispositivo presentaba la ventaja que permitía pasar el tubo produciendo menos lesiones que otros dispositivos en uso y también ayudaba a una visión más clara, ya que, podría ser utilizado como un espéculo ${ }^{26}$.

El Dr. Magill recibió el título de sir de manos de la Reina en 1960 y falleció a los 98 años de edad.

Como anécdota, en 1920 la Queen's University rechazó su tesis, la cual se basaba en la anestesia endotraqueal, ya que se le consideró de "poco valor".

\section{Ringer, Sidney (1835-1910)}

Nació en Norwich en 1835, Inglaterra. A los 20 años de edad obtuvo un puesto como aprendiz jun- to a un doctor local, algo habitual en esa época como preludio a la carrera de medicina. Luego, logró estudiar gracias al apoyo económico de parientes y conocidos, graduándose en 1860 en el University College de Londres. Posteriormente, trabajó por cortos períodos en diversos lugares, entre ellos en el University College Children's Hospital y en el Great Ormond Street Children's Hospital, para finalmente retornar al hospital donde se formó dedicándose a la asistencia, docencia y especialmente a la investigación en su laboratorio $^{27}$. El Dr. Ringer fue uno de los primeros farmacólogos científico-clínico y en 1869 publicó su "Handbook of Therapeutics" el cual alcanzó un gran éxito, con numerosas ediciones y traducciones a diversos idiomas.

Su paso a la historia de la Medicina se debe a sus investigaciones referente a la influencia de los constituyentes sanguíneos sobre la contractibilidad cardíaca ${ }^{28}$. Es conocido que para muchos de sus diseños experimentales empleaba preparaciones de corazón de rana a la cual adicionaba diversas soluciones basadas en cloruro de sodio al 0,75\%. Según se relata en una ocasión ante la ausencia de su asistente de laboratorio, Ringer tuvo que preparar el mismo su solución fisiológica ("agua destilada" a la que agregaba sodio), no obstante, a diferencia de las ocasiones previas el corazón latió solo por unos pocos minutos. ¿A qué se debió esto? Resultó que su ayudante estaba empleando agua de grifo, suministrada por New River Water Company, en vez de destilada, para originar la solución "salina" de perfusión. Un cuidadoso análisis reveló que el agua en Londres en aquella época contenía una gran cantidad de calcio ${ }^{29}$. Todas las observaciones sobre el rol del calcio en la contracción cardíaca y la descripción de la solución epónima fueron publicadas en el Journal of Physiology entre 1875 y 1895 . De este modo pudo demostrar la importancia de las sales inorgánicas en los organismos ${ }^{30}$.

Sydney Ringer murió en 1910, a causa de un accidente cerebro vascular.

En 1932, el pediatra Alexis Hartmann modificó la solución de Ringer añadiéndole lactato de sodio, con el fin de evitar el desarrollo de acidosis en lactantes deshidratados por diarrea, dando lugar al suero que usamos en la actualidad ${ }^{31}$.

\section{Yankauer, Sidney (1872-1932)}

Nacido en Nueva York e hijo de padres inmigrantes alemanes judíos fue una promesa académica desde temprana edad. Tras seguir una educación conforme al ejemplo del "sueño americano" obtuvo su grado de médico el año 1893 en el colegio de Médicos y Cirujanos de Nueva York. Se desempeñó desde su internado y posteriormente durante gran parte de su carrera 
como cirujano en el Hospital Mount Sinaí, lugar que constituyó un ambiente muy estimulante para la naturaleza inventiva de Yankauer.

En cuanto a su carrera quirúrgica, desde sus comienzos fue progresivamente especializándose en enfermedades de oídos, nariz y laringe. Para la época, la otorrinolaringología estaba dando sus primeros pasos, por lo que el Dr. Yankauer jugó un rol fundamental en el desarrollo de esta rama.

Yankauer destacó sin duda por ser un inventor prolífico, siendo algunas de sus invenciones no relacionadas con el quehacer médico. Sin embargo, su dispositivo de succión se inmortalizó en la historia de la medicina. Este fue inicialmente pensado para mantener el campo quirúrgico limpio durante la tonsilectomía, para luego transformarse en una pieza maestra del diseño cuyo uso es actualmente de carácter universal. Su punta roma que previene el daño de los tejidos blandos y el diseño de su mango elegante y ángulo jamás han sido superados ${ }^{32}$.

El Dr. Yankauer contribuyó con numerosas publicaciones científicas, abarcando una amplia temática otorrinolaringológica. Su primera comunicación sobre broncoscopia fue en $1905^{33}$, solo 8 años después de la descripción de la técnica. Pero también publicó sobre casos tan exóticos como sanguijuelas traqueales ${ }^{34}$ y la descripción de la trompa de Eustaquio en el oso hormiguero ${ }^{35}$.

Es sorprendente que la descripción original del dispositivo de succión de Yankauer (ca. 1907) parece no haber sido publicada.

\section{Doppler, Christian A. (1803-1853)}

Christian Doppler nació en Salzburgo, Austria. Provenía de una rica familia dedicada a la cantería. Se graduó en 1829 en la Universidad de Viena en matemáticas superiores, mecánica y astronomía. Luego tomó, en la misma universidad, una ayudantía en una cátedra de matemáticas, por un tiempo que se prolongó demasiado para sus intereses, por lo que solicitó en muchas ciudades un trabajo permanente, siendo en todos ellas rechazado y no pudiendo así asegurar una posición académica. Debido a esto, tuvo que emplearse como contador en una empresa de hilado de algodón, para luego de dos años decidir emigrar hacia Estados Unidos de América. Circunstancialmente, le fue ofrecido un cargo docente en la Escuela Técnica Secundaria de Praga, siendo contratado en 1836 para impartir unas pocas horas de clases de matemáticas a la semana. Finalmente, logró un cargo de profesor a horario completo en el Instituto Politécnico de Viena a los 38 años de edad. Fue aquí, en 1842, cuando presentó en un congreso de ciencias naturales en Praga su descubri- miento (a futuro, efecto Doppler) el cual le haría pasar a la historia ${ }^{36}$. La comunicación, basada simplemente en la observación de fenómenos naturales, se titulaba "Über das farbige Licht der Doppelsterne und einiger anderer gestirne des Himmels" (Sobre la luz coloreada de las estrellas dobles y algunas otras estrellas del cielo) ${ }^{37}$. Doppler quiso aplicar inicialmente su principio a la observación astronómica.

La primera prueba experimental de la validez del efecto Doppler para las ondas sonoras, fue efectuado en el año 1845 por el meteorólogo holandés Christoph Hendrik Diederik Buys Ballot mediante una original e ingeniosa demostración: instaló a un grupo de músicos trompetistas en un vagón descubierto de un tren en marcha, tocando siempre la misma nota al unísono. Al mismo tiempo colocó a un grupo de observadores situados a lo largo de la vía, registrando qué tono estimaban según el tren se acercaba o alejaba (resultado: más agudo de cerca y más grave de lejos). Posteriormente, Doppler condujo un experimento similar, actualizando su nueva evidencia ${ }^{38}$.

Aunque su actividad científica era exitosa, su rol como docente fue más problemático; así en 1844 sus alumnos lo denunciaron por ser demasiado exigente en los exámenes, y fue apartado de la docencia, motivándolo a buscar nuevamente trabajo, aceptando la cátedra de física, matemáticas y mecánica en la Academia de Minas y Forestal de Banska Stiavnica. Hacia el año 1850 fue nombrado director del Instituto de Física de la Universidad Imperial de Viena, alcanzando el pináculo de su carrera (Allí reprobó a un joven científico llamado Gregor Mendel). No obstante, ya presentaba graves problemas con la tuberculosis, falleciendo a los 49 años en Venecia ${ }^{38}$.

Un siglo después Satumora y cols. ${ }^{39}$ fueron los primeros en aplicar el efecto Doppler en la medicina.

\section{Charrière, Joseph-Frédéric-Benoît (1803-1876)}

La escala francesa (en inglés: French) o escala de Charrière, es una medida que se utiliza para expresar el calibre de diferentes instrumentos médicos (sondas, catéteres, dilatadores, etc.), la cual fue creada en 1842 por el cuchillero y fabricante de instrumentos médicos Joseph-Benoît Charrière ${ }^{40}$. Principalmente en países europeos se utiliza la expresión "Charrière", mientras que en otros se emplea la palabra French. En la práctica médica actual es un estándar internacional y casi universal, conocido como sistema French ${ }^{41}$.

Joseph-Frédéric-Benoît Charrièrre nació en Suiza en el año de 1803, siendo criado por su abuelo durante su infancia. A la edad de 13 años es llevado a Paris para aprender el oficio de cuchillero, donde a los 17 años queda a cargo del taller debido a la muerte de su padre. 
Charrièrre se especializó en la fabricación de instrumental quirúrgico, alcanzando gran fama por la alta calidad de sus productos, siendo estos solicitados por connotados cirujanos a requerimiento personal. Dentro de los cuales destacó el Dr. Guillaume Dupuytren, quién fue su mentor y mecenas y quién lo ayudó a lograr su desarrollo profesional, como también el reconocimiento de su empresa a nivel mundial ${ }^{42}$.

Además, del legado de su sistema de medición del calibre, destacó en el diseño y producción de numeroso e innovador instrumental quirúrgico, especialmente en el área de la urología, como también tuvo participación en el desarrollo de la jeringa moderna ${ }^{43}$.

Charrièrre murió en 1873 en Paris y se encuentra sepultado en el cementerio de Montparnasse.

\section{Discusión}

Los epónimos médicos son muchísimos, estimándose en más de ocho mil ${ }^{44}$, y aunque muchos de ellos ya están fuera de uso, es probable que algunos seguirán estando vigentes en nuestro lenguaje médico ${ }^{2,45}$.

No obstante, son términos sin raíz etimológicas y se debe tener presente que su uso en ocasiones es una fuente de confusión, además pueden ser carentes de rigurosidad científica y presentar un carácter etnocéntrico y androcéntrico. Asimismo, a menudo no reflejan el trabajo en equipo detrás de su descubrimiento o invención, en ocasiones la suerte juega un rol mayor en su origen y puede presentar conflictos éticos, recomendándose para estos últimos su abandono ${ }^{46,47}$. Por ello, sus detractores recomiendan su cambio en favor del uso de una nomenclatura más descriptiva ${ }^{3}$.
Así entonces, aunque su uso es controversial y probablemente lo seguirá siendo, reflejan la apasionante crónica de la medicina, honran las contribuciones históricas y constituyen habitualmente un justo homenaje. Sin embargo, importantes personajes históricos en la medicina con sus trascendentes contribuciones no derivaron en uso de epónimo, a saber, Willem Einthoven y el desarrollo del electrocardiógrafo, Wilhelm Röntgen el descubrimiento de los rayos X y René Laënnec el inventor del estetoscopio.

\section{Responsabilidades éticas}

Protección de personas y animales: Los autores declaran que los procedimientos seguidos se conformaron a las normas éticas del comité de experimentación humana responsable y de acuerdo con la Asociación Médica Mundial y la Declaración de Helsinki.

Confidencialidad de los datos: Los autores declaran que han seguido los protocolos de su centro de trabajo sobre la publicación de datos de pacientes.

Derecho a la privacidad y consentimiento informado: Los autores han obtenido el consentimiento informado de los pacientes y/o sujetos referidos en el artículo. Este documento obra en poder del autor de correspondencia.

\section{Conflicto de intereses}

Los autores declaran no tener conflicto de intereses.

\section{Referencias}

1. Goic A. Sobre el uso de epónimos en medicina. Rev Med Chile. 2009;137:150810.

2. Zafra Anta MA, Medino Muñoz J, Gorrotxategi Gorrotxategi P, Ponte Hernando F. Use of eponyms in Spanish in paediatric journals over the last ten years. Is it changing?. An Pediatr (Barc). 2018;88(1):58-60.

3. Woywodt A, Matteson E. Should eponyms be abandoned? Yes. BMJ. 2007;335(7617):424

4. Fargen KM, Hoh BL. The debate over eponyms. Clin Anat. 2014;27:1137-40.

5. Aronson JK. Medical eponyms: taxonomies, natural history, and the evidence. BMJ. 2014; 349: g7586.

6. Aleixandre-Benavent R, Alonso-Arroyo A, González-Muñoz M, González de
Dios J. Comunicación científica (XXIV) Lenguaje médico (2): Los epónimos en el lenguaje médico de la pediatría. Acta Pediatr Esp. 2015;73(6):164-70.

7. Tatem AJ, Klaassen Z, Lewis RW, Terris MK. Frederick Eugene Basil Foley: his life and innovations. Urology. 2013; 81(5):927-31.

8. Zorgniotti AW. Frederic E. B. Foley. Early development of balloon catheter. Urology. 1973;1:75-80.

9. Foley FE. A hemostatic bag catheter: a one piece latex rubber structure for control of bleeding and constant drainage following prostatic resection. J Urol. 1937;38:134-9.

10. Foley F. A new plastic operation for stricture at the uretero-pelvic junction. Report of 20 operations. J Urol. 1937;38:643-72.

11. Foley FE. A completely rotatable resectoscope. J Urol. 1949;62:381-6.
12. Seldinger SI. Catheter replacement of the needle in percutaneous arteriography (a new technique). Acta Radiol. 1953;39:36876.

13. Seldinger SI. Localization of parathyroid adenomata by arteriography. Acta Radiol. 1954:42:353-66.

14. Seldinger SI. Percutaneous transhepatic cholangiography. Acta Radiol [Suppl] (Stockh) 1966;253:1-134.

15. Ward OC. John Langdon Down: the man and the message. Downs Syndr Res Pract. 1999;6:19-24.

16. Down JL. Observations of an ethnic classification of idiots. Clin Lect Rep London Hosp Rep. 1866;3:259-62.

17. Allen G, Benda CE, Böök JA, et al. Mongolism. Am J Hum Genet 1961;13:426.

18. Minassian H, Cowan SW, Yeo CJ. Robert Reynolds Macintosh, M.D. (1897- 
1989): Forefather of Anesthesiology, Advocate for Patient Safety. Am Surg. 2017;83(4):137-9.

19. Macintosh RR. A new laryngoscope. Lancet 1943;1:205.

20. Greenland KB, Eley V, Edwards MJ, Allen $\mathrm{P}$, Irwin MG. The origins of the snif ng position and the three axes alignment theory for direct laryngoscopy. Anesth Intensive Care. 2008;36(Suppl. 1):23-7.

21. Robinson DH, Toledo AH. Historical development of modern anesthesia. J Invest Surg. 2012;25(3):141-9.

22. Scott J, Baker PA. How did the Macintosh laryngoscope become so popular? Paediatr Anaesth. 2009;19(Suppl 1):24-9.

23. McLachlan G. Sir Ivan Magill KCVO, DSc, MB, BCh, BAO, FRCS, FFARCS (Hon), FFARCSI (Hon), DA, (18881986). Ulster Med J. 2008;77(3):146-52.

24. Pallister WK. Sir Ivan Whiteside Magill (1888-1986) and tracheal intubation. En: Atkinson RS \& Boulton TB. editors. The history of anaesthesia. London: Royal Society of Medicine Services Limited; 1988.

25. Liverpool Society of Anaesthetists. Demonstration of blind nasal intubation by Dr Magill. BMJ. 1932;2:838.

26. Magill I. Forceps for intratracheal anaesthesia. BMJ. 1920;2:670.

27. Ellis H. Sydney Ringer: physician, physiologist and pharmacologist. Br J Hosp Med (Lond) 2010;71(11):645

28. Ringer S. Concerning the influence exerted by each of the constituents of the blood in the contraction of the ventricle. J Physiol (London). 1882;3:380-93.
29. Ringer S. A further contribution regarding the influence of the different constituents of the blood on the contraction of the heart. J Physiol. 1883;4:29-42.

30. Ringer S, Buxton DW. Concerning the Action of small quantities of Calcium, Sodium, and Potassium Salts upon the Vitality and Function of Contractile Tissue and the Cuticular Cells of Fishes Journal of Physiology (London). 1885;6:154-61.

31. Hartmann AF, Senn MJ. Studies in the metabolism of sodium r-Lactate. III. Response of human subjects with liver damage, disturbed water and mineral balance, and renal insufficiency to the intravenous injection of sodium r-Lactate. J Clin Invest. 1932;11:345-55.

32. Smith T. Sidney Yankauer 1872-1932 the man behind the mask. En: The history of Anaesthesia Society Proceedings 25th Anniversary. Wokingham 2012, Vol 45:74-75.

33. Yankauer S. Foreign body in the bronchus: removal with the aid of the bronchoscope: recovery. Medical Record 1905; lxvii: 217.

34. Yankauer S. A leech in the trachea, report of two cases. Laryngoscope. 1904;14:567.

35. Yankauer S. The Eustachian tube of the anteater. Laryngoscope. 1904;14:656.

36. Coman IM. Christian Andreas Doppler-the man and his legacy. Eur J Echocardiogr. 2005;6:7-10.

37. Doppler C. Über das farbige Licht der Doppelsterne und einiger anderer Gestirne des Himmels, Abhandlungen der königlichen böhmischen Gesellschaft der
Wissenschaften. 1843:2:465-82.

38. Roguin A. Christian Johann Doppler: the man behind the effect. Br J Radiol. 2002;75:615-9.

39. Satumora S. Study of flow patterns in peripheral arteries by ultrasonics. J Acoust. Soc. Jpn 1959;15:151-8.

40. Osborn NK, Baron TH. The history of the 'French' gauge. Gastrointest Endosc. 2006;63(3):461-2.

41. Bowen DK, Wan J, Engel R, Lyon RP, Dielubanza E, Bloom DA. Sounds and Charrière: the rest of the story. J Pediatr Urol. 2014;10(6):1106-10.

42. Badawi JK. Joseph-Frédéric-Benoît Charrière - How to Explain His Success as One of the Most Famous Surgical Instrument Makers Regarding His Life from Childhood to Death. Urol Int. 2017;98(2):236-40.

43. Iserson KV. J.-F.-B. Charrière: the man behind the "French" gauge. J Emerg Med. 1987;5(6):545-8.

44. Whonamedit.com. Accedido el día 25 de septiembre de 2017. Disponible en: http:// www.whonamedit.com/.

45. Whitworth JA. Should eponyms be abandoned? No. BMJ. 2007;335(7617):425.

46. Harper PS. Naming of syndromes and unethical activities: the case of Hallervorden and Spatz. Lancet. 1996;348(9036):1224-5.

47. Wallace DJ, Weisman M. Should a war criminal be rewarded with eponymous distinction? the double life of Hans Reiter (1881-1969). J Clin Rheumatol. 2000;6:49-54. 